\title{
Dark and grey compressional dispersive Alfven solitons in plasmas
}

\author{
P.K. Shukla, B. Eliasson and Lennart Stenflo
}

\section{Linköping University Post Print}

N.B.: When citing this work, cite the original article.

Original Publication:

P.K. Shukla, B. Eliasson and Lennart Stenflo, Dark and grey compressional dispersive Alfven solitons in plasmas, 2011, Physics of Plasmas, (18), 6, 064511.

http://dx.doi.org/10.1063/1.3602463

Copyright: American Institute of Physics http://www.aip.org/

Postprint available at: Linköping University Electronic Press http://urn.kb.se/resolve?urn=urn:nbn:se:liu:diva-69872 


\title{
Dark and grey compressional dispersive Alfvén solitons in plasmas
}

\author{
P. K. Shukla, ${ }^{1, a)}$ B. Eliasson, ${ }^{1, b)}$ and L. Stenflo ${ }^{2, c)}$ \\ ${ }^{1}$ International Centre for Advanced Studies in Physical Sciences, Faculty of Physics and Astronomy, \\ Ruhr University Bochum, D-44780 Bochum, Germany \\ ${ }^{2}$ Department of Physics, Linköping University, SE-58183 Linköping, Sweden
}

(Received 15 April 2011; accepted 3 June 2011; published online 30 June 2011)

The amplitude modulation of compressional dispersive Alfvén (CDA) waves in a low- $\beta$ plasma is considered. It is shown that the dynamics of modulated CDA waves is governed by a cubic nonlinear Schrödinger equation, which depicts the formation of a dark/grey envelope CDA soliton.

(c) 2011 American Institute of Physics. [doi:10.1063/1.3602463]

The nonlinear propagation of hydromagnetic waves is of significant interest in understanding the properties of intrinsic localized excitations of electromagnetic disturbances in magnetized space and laboratory plasmas. ${ }^{1}$ Hydromagnetic waves include the Alfvén waves propagating along and across the external magnetic field direction, as well as the coupled fast and slow Alfvén waves and the kinetic and inertial Alfvén waves ${ }^{2}$ that propagate obliquely to the magnetic field. Dispersive hydromagnetic waves, which can be parametrically excited, ${ }^{3}$ have been used for heating ions and electrons in plasmas.

Our low-frequency compressional dispersive Alfvén (CDA) wave propagates here across a homogeneous magnetic field $\left(\widehat{\mathbf{z}} B_{0}\right)$, where $\widehat{\mathbf{z}}$ is the unit vector along the $z$-axis in a Cartesian coordinate system and $B_{0}$ the strength of the magnetic field in a uniform magnetoplasma. The restoring force on the CDA wave comes from the wave magnetic pressure, and the ion mass provides inertia to sustain the CDA wave. The CDA wave dispersion arises due to the electron polarization drift in the wave electric field $\mathbf{E}_{\perp}=\widehat{\mathbf{x}} E_{x}+\widehat{\mathbf{y}} E_{y}$, where $\widehat{\mathbf{x}}$ and $\widehat{\mathbf{y}}$ are the unit vectors along the $x$ and $y$ axes, respectively. In a quasi-neutral plasma, the $x$ components of the electron and ion fluid velocities are identical, but the $x$ and $y$ components of the electron fluid velocities differ owing to the electron polarization. The electrons carry current only along the $y$-direction. The CDA wave compresses the magnetic field-lines without bending them.

The nonlinear propagation of one-dimensional CDA waves along the $x$-axis is governed by the ion continuity equation

$$
\frac{d n}{d t}+n \frac{\partial u}{\partial x}=0
$$

the ion momentum equation

$$
\frac{d u}{d t}+\frac{V_{A}^{2}}{2 n} \frac{\partial}{\partial x}\left(B^{2}+2 \beta \ln n\right)=0,
$$

and Faraday's law of electromagnetic induction

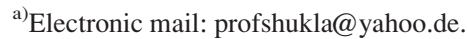

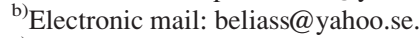

${ }^{c)}$ Electronic mail: lennart.stenflo@physics.umu.se.
}

$$
\frac{d}{d t}\left(1-\lambda_{e}^{2} \frac{\partial^{2}}{\partial x^{2}}\right) B+B \frac{\partial u}{\partial x}=0,
$$

where $d / d t=(\partial / \partial t)+u \partial / \partial x, n$ is the normalized (by the equilibrium density $n_{0}$ ) ion number density, $u$ the $x$ - component of the ion fluid velocity, $B$ the normalized (by $B_{0}$ ) compressional (along $\widehat{\mathbf{z}}$ ) magnetic field, $V_{A}=B_{0} /\left(4 \pi n_{0} m_{i}\right)^{1 / 2}$ the Alfvén speed, $m_{i}$ the ion mass, $\beta=C_{s}^{2} / V_{A}^{2}$ the plasma beta, $C_{s}$ the ion sound speed, $\lambda_{e}=c / \omega_{p e}$ the electron skin depth, $c$ the speed of light in vacuum, $\omega_{p e}=\left(4 \pi n_{0} e^{2} / m_{e}\right)^{1 / 2}$ the electron plasma frequency, $e$ the magnitude of the electron charge, and $m_{e}$ the electron mass. It should be noted that in deriving (2) and (3), we used the electric field from the electron momentum equation, while (2) also employed Ampère's law, which is valid for $\partial / \partial t \ll c \partial / \partial x$. The $\lambda_{e}$-term in (3) comes from the electron inertia.

Letting $\quad n=1+N_{1} \quad$ and $B=1+b_{1}, \quad$ where $N_{1}=n_{1} / n_{0} \ll 1$ and $b_{1}=B_{1} / B_{0} \ll 1$, we linearize (1)-(3) and combine the resultant equations to obtain the wave equation

$$
\left(1-\lambda_{e}^{2} \frac{\partial^{2}}{\partial x^{2}}\right)\left(\frac{\partial^{2}}{\partial t^{2}}-C_{s}^{2} \frac{\partial^{2}}{\partial x^{2}}\right) b_{1}-V_{A}^{2} \frac{\partial^{2} b_{1}}{\partial x^{2}}=0,
$$

which can be Fourier transformed by supposing that $b_{1}$ is proportional to $\exp (-i \omega t+i k x)$, where the frequency $\omega$ and the wavenumber are related by ${ }^{4}$

$$
\omega^{2}=k^{2} C_{s}^{2}+\frac{k^{2} V_{A}^{2}}{\left(1+k^{2} \lambda_{e}^{2}\right)} .
$$

We note that for $k^{2} \lambda_{e}^{2} \gg 1$, Eq. (5) gives $\omega^{2}=k^{2} C_{s}^{2}+\omega_{L H}^{2}$, where $\omega_{L H}=\sqrt{\omega_{c e} \omega_{c i}}$ is the lower-hybrid resonance frequency. Here, we have denoted $\omega_{c e}=e B_{0} / m_{e} c$ and $\omega_{c i}=e B_{0} / m_{i} c$ as the electron and ion gyrofrequencies, respectively.

Several comments are in order. First, in the past, many authors $^{5-7}$ discussed non-envelope soliton ${ }^{5,6}$ and cnoidal wave $^{7}$ solutions of the CDA waves based on Eqs. (1)-(3) without the $\beta$-term in (2). Second, without the electron inertia induced dispersion and finite $\beta$ effects, Eqs. (2) and (3) 
admit self-similar solutions in the form of simple waves and the formation of shocks. ${ }^{8}$ Third, ignoring the electron inertial effect, Shukla et al. ${ }^{9}$ examined the effect of finite $\beta$ on the shock structures. Fourth, the combined influence of dispersion and dissipation on non-stationary CDA waves in a zero$\beta$ plasma has been examined by Chakrabarti et al ${ }^{10}$ Finally, when $\partial\left(B^{2}+2 \beta \ln n\right) / \partial x=0$ in Eq. (2), we end up obtaining the solution of Eqs. (1) and (2) in the form ${ }^{11}$ $n(t, x)=n_{0}(\xi) /\left[1+t u_{0}^{\prime}(\xi)\right]$ and $u(t, x)=u_{0}(\xi)$, where $n_{0}$ is the initial density profile, $u_{0}$ the initial velocity $\xi=x-u t$, and the prime denotes the differentiation with respect to $\xi$. It turns out that for this particular case, the ion density blows up and becomes infinite if $u_{0}^{\prime}(\xi)$ is negative at time $t^{*}=-1 / u_{0}^{\prime}\left(\xi^{*}\right)$, where $\xi^{*}$ is the value of $\xi$ where $u_{0}^{\prime}(\xi)$ has its largest negative value.

In the following, we consider the amplitude modulation of a finite amplitude CDA pump with the magnetic field $B_{1} \exp \left(-i \omega_{0} t+i k_{0} x\right)+$ complex conjugate, where $B_{1}$ is the wave magnetic field perturbation along the $z$-axis and $\omega_{0}=k_{0} V_{A} /\left(1+k_{0}^{2} \lambda_{e}^{2}\right)^{1 / 2}$ for $\beta \ll 1$. The nonlinear interaction between the CDA pump and quasi-stationary compressional magnetic field perturbations give rise to a slowly varying envelope of CDA waves whose magnetic field $\mathcal{B}$ varies slowly on the temporal-spatioscales $(T, X)$. The evolution of the CDA envelope is given by

$$
i\left(\frac{\partial}{\partial T}+V_{g} \frac{\partial}{\partial X}\right) \mathcal{B}+P \frac{\partial^{2} \mathcal{B}}{\partial X^{2}}-\omega_{0} B_{s} \mathcal{B}=0
$$

where $\partial \mathcal{B} / \partial T \ll \omega_{0} \mathcal{B}, \quad V_{g}=\partial \omega_{0} / \partial k_{0}=V_{A} /\left(1+k_{0} \lambda_{e}^{2}\right)^{3 / 2}$ is the group velocity of the pump, $P=(1 / 2) \partial V_{g} / \partial k_{0}$ $=-3 \omega_{0} \lambda_{e}^{2} / 2\left(1+k_{0}^{2} \lambda_{e}^{2}\right)^{2} \equiv-P_{0}$ represents the pump wave group dispersion $B_{s}=B_{1 s} / B_{0}$, and $B_{1 s}\left(\ll B_{0}\right)$ the compressional magnetic field perturbation associated with a quasi-stationary plasma slow motion. We note that (6) has been deduced from (1)-(3) in the WKB approximation. ${ }^{12}$

The expression for $B_{s}$ is determined by averaging the inertialess ion momentum Equation (2) over $2 \pi / \omega_{0}$. We have

$$
\left\langle V_{i} \frac{\partial V_{i}}{\partial X}\right\rangle=-V_{A}^{2} \frac{\partial B_{s}}{\partial X}-\frac{V_{A}^{2}}{2 B_{0}^{2}} \frac{\partial\left\langle\mathcal{B}^{2}\right\rangle}{\partial X}
$$

where the angular bracket denotes an ensemble average over the CDA wave-period, and the $x$-component of the ion fluid velocity in the CDA wave magnetic field is $V_{i}$ $=\left(1+k_{0}^{2} \lambda_{e}^{2}\right)^{1 / 2} V_{A} \mathcal{B} / B_{0}$. Accordingly, Eq. (7) yields

$$
B_{s}=-\frac{\left(2+k_{0}^{2} \lambda_{e}^{2}\right)}{2 B_{0}^{2}}|\mathcal{B}|^{2} .
$$

Substituting for $B_{s}$ from (8) into (6) we obtain the cubic nonlinear Schrödinger equation

$$
i\left(\frac{\partial}{\partial T}+V_{g} \frac{\partial}{\partial X}\right) \mathcal{B}-P_{0} \frac{\partial^{2} \mathcal{B}}{\partial X^{2}}+Q|\mathcal{B}|^{2} \mathcal{B}=0,
$$

where $Q=\omega_{0}\left(2+k_{0}^{2} \lambda_{e}^{2}\right) / 2 B_{0}^{2}$. Since the product of the group dispersion $-P_{0}$ and the coefficient of nonlinearity $Q$ is negative, a finite amplitude CDA pump is stable against quasi-stationary magnetic perturbations.

Possible stationary solutions of Eq. (9) are the dark and grey envelope solitons. ${ }^{13,14}$ The profile of the dark envelope soliton is

$$
\mathcal{B}=\sqrt{D(\xi, T)} \exp (i \Theta),
$$

where $\xi=X-V_{g} T$, and

$$
D(\xi, T)=D_{0}\left[1-\operatorname{sech}^{2}\left(\xi / L_{d}\right)\right]=D_{0} \tanh ^{2}\left(\xi / L_{d}\right),
$$

where $\Theta=\left(1 / 2 P_{0}\right)\left[V_{g} X-\left(0.5 V_{g}^{2}-2 P_{0} Q D_{0}^{2}\right) T\right]$ is a phasor, $L_{d}=\sqrt{2 P_{0} / Q D_{0}}$ the soliton pulse width, and $D_{0}$ is the value of $D$ as $|\xi| \rightarrow \infty$. Furthermore, the profile of the grey envelope soliton is

$$
\mathcal{B}=\sqrt{G(\xi, T)} \exp (i \Theta),
$$

where

$$
G(\xi, T)=G_{0}\left[1-H^{2} \operatorname{sech}^{2}\left(\xi / L_{g}\right)\right]
$$

with the soliton pulse width $L_{g}=(1 / H) \sqrt{2 P_{0} / Q G_{0}}$. Here $H$ is a dimensionless parameter, representing the modulation depth $(0<H \leq 1)$. It is given by $H^{2}=1+\left(V_{g}-V_{0}\right)^{2} /$ $2 P_{0} Q G_{0}$, where $V_{0}$ is an independent real constant, satisfying the condition $V_{0}-\sqrt{2 P_{0} Q G_{0}^{2}} \leq V_{g} \leq V_{0}+\sqrt{2 P_{0} Q G_{0}^{2}}$. For $V_{0}=V_{g}$, we have $H=1$ and thus recover the dark soliton. Both dark and grey envelope solitons represent a localized region of negative wave magnetic density propagating with the group velocity $V_{g}$. Phases inside the envelope solitons oscillate rapidly.

In this Brief Communication, we have considered the amplitude modulation of the CDA waves against quasi-stationary magnetic field perturbations in a plasma with $\beta \ll 1$. It is found that the modulated CDA wave packet remains stable and it propagates in the form of a dark/grey envelope soliton. The latter is composed of a localized region of negative magnetic wave density that is trapped in a self-created magnetic field depression. The present result may help to interpret experimental data which have a signature of localized compressional magnetic field perturbations in very low$\beta$ magnetized space and laboratory plasmas.

This research was supported by the Deutsche Forschungsgemeinschaft through the project $\mathrm{SH} 21 / 3-1$ of the Research Unit 1048.

\footnotetext{
${ }^{1}$ A. Hasegawa and C. Uberoi, The Alfvén Wave (National Technical Information Service, US Department of Commerce, Springfield, Virginia, 1982).

${ }^{2}$ R. P. Sharma and P. K. Shukla, Phys. Fluids 26, 87 (1983).

${ }^{3}$ G. Murtaza and P. K. Shukla, J. Plasma Phys. 31, 423 (1984); P. K. Shukla and L. Stenflo, Phys. Scr. T60, 32 (1995).

${ }^{4}$ B. B. Kadomtsev, Cooperative Effects in Plasmas (in review), edited by V. D. Shafranov (Kluwer Academic/Plenum, New York, 2001), pp. 189-192.

${ }^{5}$ J. H. Adlam and J. E. Allen, Philos. Mag. 3, 448 (1958).
} 
${ }^{6}$ D. A. Tidman and N. A. Krall, Shock Waves in Collisionless Plasmas (John Wiley \& Sons, New York, 1971), and references therein.

${ }^{7}$ C. M. C. Nairn, R. Bingham, and J. E. Allen, J. Plasma Phys. 71, 631 (2005).

${ }^{8}$ L. Stenflo, A. B. Shvartsburg, and J. Weiland, Phys. Lett. A 225, 113 (1997).

${ }^{9}$ P. K. Shukla, B. Eliasson, M. Marklund, and R. Bingham, Phys. Plasmas 11, 2311 (2004).
${ }^{10}$ N. Chakrabarti, C. Maity, and H. Schamel, Phys. Rev. Lett. 106, 145003 (2011).

${ }^{11}$ G. B. Whitham, Linear and Nonlinear Waves (John Wiley \& Sons, New York, 1974).

${ }^{12}$ V. I. Karpman and E. M. Krushkal, Zh. Eksp. Teor. Fiz. 55, 530 (1968) [Sov. Phys. JETP 28, 277 (1969)].

${ }^{13} \mathrm{~A}$. Hasegawa, Plasma Instabilities and Nonlinear Effects (Springer-Verlag, Berlin, 1975), p. 199

${ }^{14}$ R. Fedele, Phys. Scr. 65, 502 (2002). 